\title{
O uso da Língua Brasileira de Sinais para a promoção da inclusão em museus: revisando a literatura
}

\section{The use of brazilian sign language to promote inclusion in museums: reviewing the literature}

\footnotetext{
(iD) Gabriela Sehnem Heck Grande do Sul, Brasil heck.gs@gmail.com

Resumo: Este trabalho teve como objetivo principal compreender como a elaboração e execução de atividades específicas em museus, voltadas à comunidade surda, podem ser aliadas para a inclusão destes visitantes. Para a coleta de dados, foi realizada uma pesquisa bibliográfica a partir dos bancos de dados on-line BDTD-IBICT e Google Acadêmico, abrangendo os anos de 2009 e 2019. Os resultados obtidos foram divididos em três eixos: recursos humanos, recursos estruturais e recursos visuais. Conclui-se que é indispensável a presença de mediadores e funcionários surdos como condição para a elaboração de atividades, mas também a presença de pessoal capacitado, surdos ou ouvintes, para a realização da tradução e interpretação. Cabe às instituições promoverem atividades de formação e 


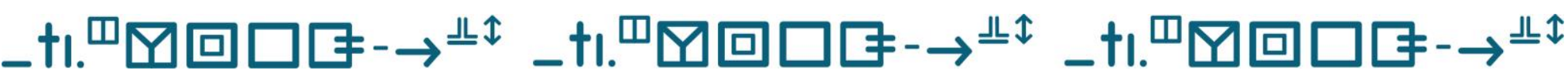

O uso da Língua Brasileira de Sinais para a promoção da inclusão em museus: revisando a literatura

Gabriela Sehnem Heck • José Luis Schifino Ferraro

capacitação voltadas aos funcionários e à comunidade em geral, ampliando a acessibilidade. Sugere-se a criação de uma coordenadoria específica para assuntos que envolvam a acessibilidade e inclusão, permitindo o desenvolvimento de atividades para os surdos.

Palavras-chave: Língua Brasileira de Sinais- Libras. Museu. Inclusão. Surdos.

Abstract: This work's main goal was to understand how the elaboration and execution of specific activities in museums focused on the deaf community could be aligned for the inclusion of these visitors. For data collection, a bibliographic search was carried out using the online databases BDTDIBICT and Google Scholar, covering the years 2009 and 2019. The results obtained were divided into three axes: human resources, structural resources and visual aids. The conclusion is that the presence of mediators and deaf employees is essential as a condition for the elaboration of activities, but also the presence of trained personnel capable of translating and interpreting elements. Institutions should organize and provide specialized training activities aimed at employees and the general community, expanding accessibility. It is suggested to create a specific coordination to deal with issues involving accessibility and inclusion, allowing the development of meaningful and specialized activities for the Deaf.

Keywords: Brazilian Sign Language-Libras. Museum. Inclusion. Deaf. 


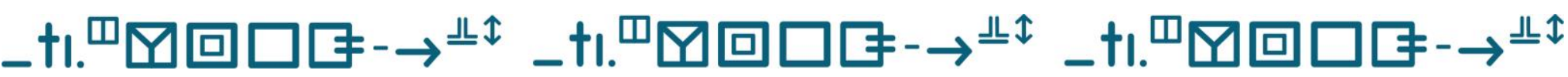

O uso da Língua Brasileira de Sinais para a promoção da inclusão em museus: revisando a literatura

Gabriela Sehnem Heck • José Luis Schifino Ferraro

\section{Introdução}

A língua brasileira de sinais (Libras) corresponde a uma "língua natural adquirida de forma espontânea pela pessoa surda em contato com [outras] pessoas que usam essa língua" (QUADROS, 1997, p. 27). Dessa forma, os surdos têm, em geral, a aquisição da Libras como primeira língua (L1), desenvolvendo o Português como segunda língua (L2). Além disso, membros da comunidade surda se reconhecem por meio de uma identidade que é compartilhada pelas demais pessoas que utilizam a língua de sinais (BISOL; SPERB, 2010).

A utilização da Libras é uma das formas mais eficazes de se incluir a pessoa surda em todos os espaços, porém, sua utilização, exclusivamente, não garante a acessibilidade. Em geral, é necessário um complemento visual, articulando os sinais a imagens, textos, vídeos, fotos, entre outros recursos (GOMES; SOUZA; SOARES, 2015). Da mesma forma, a utilização de somente recursos visuais, sem o auxílio da Libras, não garante o acesso completo à informação.

Ao nos referirmos aos espaços de inclusão, a utilização de museus vem se destacando por seu significativo apelo visual, que contribui para a promoção de experiências multimodais e sensoriais, permitindo uma comunicação mais eficiente com o público (GOMES; SOUZA; SOARES, 2015). Ao longo do século $X X$, diferentes demandas surgiram para ser atendidas pelos museus, o que permitiu a formação de um novo público, gerando a necessidade de novas ações de 


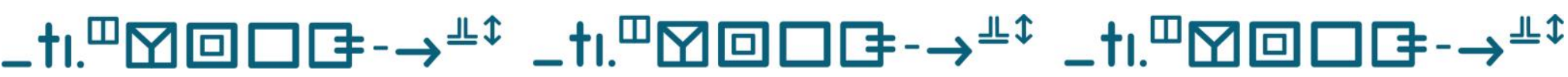

O uso da Língua Brasileira de Sinais para a promoção da inclusão em museus: revisando a literatura

Gabriela Sehnem Heck • José Luis Schifino Ferraro

perfis de atendimento e desenvolvimento de programas voltados às necessidades específicas destes, como também a adaptação da linguagem e dos meios de comunicação nesses espaços (OLIVEIRA, 2015).

Para o público surdo visitante, é sugerido que haja a associação entre a Libras e as imagens e textos, porém não se recomenda expor quantidades excessivas de informações escritas, bem como o excesso de definições, conceitos e significados, pois pode levar a uma falta de clareza na compreensão dos conceitos, coesão e itemização na linguagem (CHALHUB; BENCHIMOL; ROCHA, 2015). Além disso, a atuação de funcionários capacitados em Libras, seus tradutores e/ou intérpretes, são fundamentais para a inclusão do visitante surdo.

Silva, Rojas e Teixeira (2015, p. 106) afirmam que a "comunidade surda que procura uma visita mediada deseja acesso à informação e à comunicação. Trata-se de um processo dos surdos cidadãos de conquistar plena participação nas atividades culturais, mas encontram barreiras com a exclusão de sua língua". A falta de inclusão não permite a participação efetiva dessa parcela da sociedade, contrariando o Art. 42 da Lei $n^{\circ} 13.146$ de 06 de julho de 2015 da Lei Brasileira de Inclusão da Pessoa com Deficiência, que define que a "pessoa com deficiência tem direito à cultura, ao esporte, ao turismo e ao lazer em igualdade de oportunidades com as demais pessoas [...]" (BRASIL, 2015). A lei também Ihes garante acesso a: 


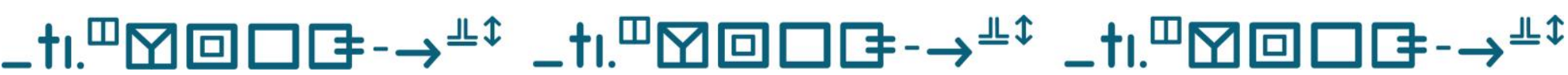

O uso da Língua Brasileira de Sinais para a promoção da inclusão em museus: revisando a literatura

Gabriela Sehnem Heck • José Luis Schifino Ferraro

| - a bens culturais em formato acessível; || - a programas de televisão, cinema, teatro e outras atividades culturais e desportivas em formato acessível; e III - a monumentos e locais de importância cultural e a espaços que ofereçam serviços ou eventos culturais e esportivos (BRASIL, 2015, Art. 42).

Dessa forma, acreditamos que a elaboração e execução de atividades específicas nos museus - voltadas à comunidade surda - podem ser importantes aliadas para a inclusão destes visitantes, usuários da Libras. Fora do Brasil, existem experiências significativas em espaços culturais que corroboram o processo de inclusão de pessoas com deficiência, em especial dos surdos (OLIVEIRA, 2015). Assim, objetivou-se a produção da presente revisão de literatura, que aponta não apenas para o conhecimento, mas para a tomada de consciência em relação ao que pode ser desenvolvido para os surdos no interior dos espaços museais, contribuindo, assim, para um maior comprometimento relacionado à inclusão do sujeito surdo.

\section{Metodologia}

Com o objetivo de compreender como são realizadas atividades em Libras nos diferentes contextos museais do Brasil, realizou-se uma revisão de literatura abrangendo os anos de 2009 e 2019 em plataformas online de trabalhos acadêmicos. Utilizou- 


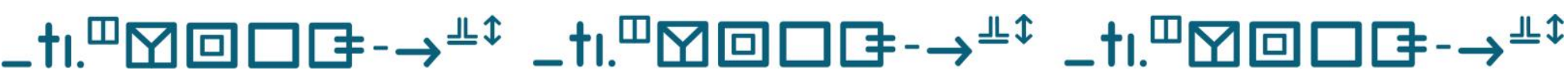

O uso da Língua Brasileira de Sinais para a promoção da inclusão em museus: revisando a literatura

Gabriela Sehnem Heck • José Luis Schifino Ferraro

se os descritores "Museu" + "Língua brasileira de sinais e Atividades" + "Língua brasileira de sinais", selecionando somente os trabalhos desenvolvidos em museus.

Na plataforma Google Acadêmico, realizou-se uma busca com as palavras-chave "Museu" + "Língua brasileira de sinais", adicionando o filtro de 10 anos, de 2009 a 2019, sem incluir patentes e citações. Obtiveram-se 725 resultados. Destes, 45 foram selecionados pelo título e, após a leitura dos resumos, 28 documentos foram escolhidos para uma análise mais profunda. Ao final, foram considerados 14 documentos que continham o foco do estudo.

Na Biblioteca Digital Brasileira de Teses e Dissertações (BDTD IBICT), utilizou-se a busca avançada, buscando por "Todos os campos: Museu" e "Todos os campos: Língua brasileira de sinais", considerando todos os termos, e obteve-se um total de 8 documentos. As pesquisas escolhidas para fazer parte da revisão foram selecionadas pelo título e, posteriormente, pelo resumo, resultando em dois documentos. Destes, um já havia sido contabilizado na plataforma Google Acadêmico.

O total obtido foi de 15 documentos, incluindo artigos, trabalhos apresentados em congresso, dissertações, monografias e um memorial histórico-narrativo (Gráfico 1). 


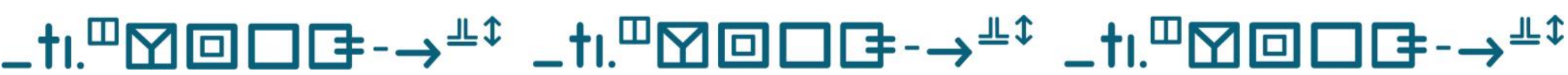

O uso da Língua Brasileira de Sinais para a promoção da inclusão em museus: revisando a literatura

Gabriela Sehnem Heck • José Luis Schifino Ferraro

Gráfico 1 - Documentos obtidos pela busca em bases de trabalhos acadêmicos

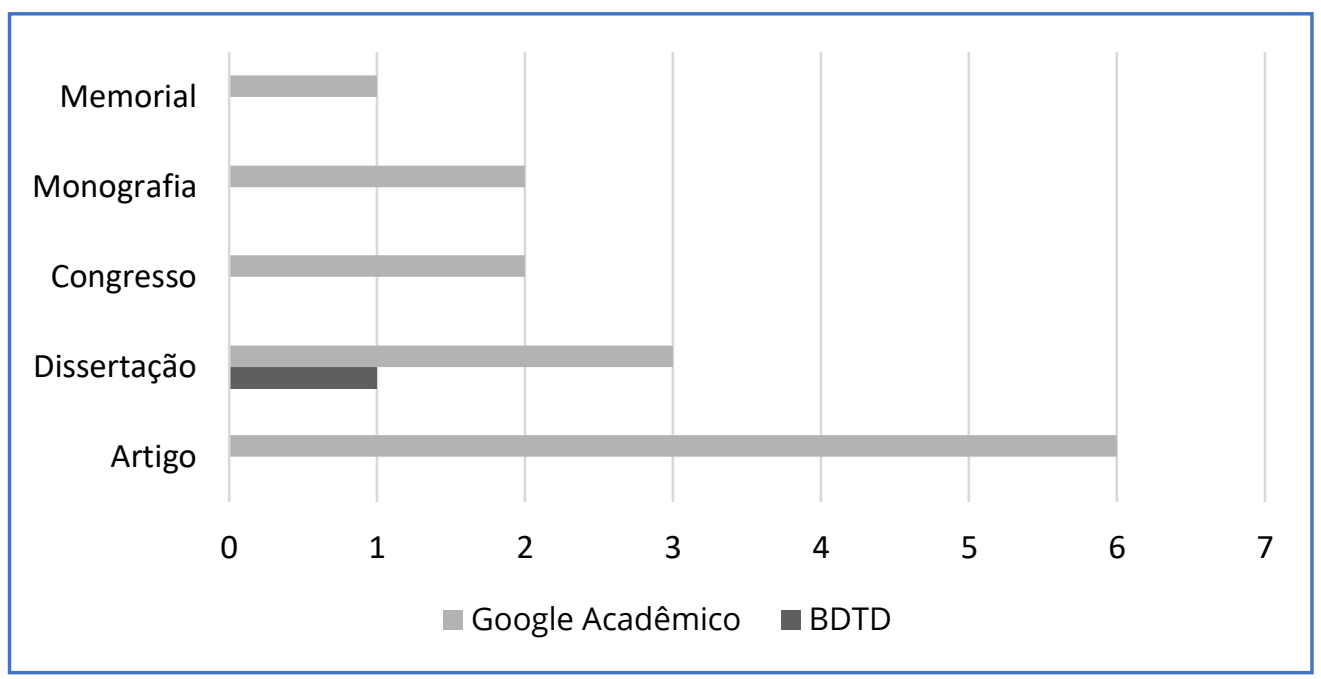

Fonte: Os autores (2020).

Descrição da imagem: Gráfico informativo sobre o percentual de documentos encontrados em bases de trabalhos acadêmicos, elaborado pelos autores, com um memorial, duas monografias, dois trabalhos de congresso, quatro dissertações e seis artigos.

As quatro dissertações consideradas nesta revisão foram escritas nos estados de São Paulo, Rio de Janeiro e Minas Gerais - região Sudeste (OLIVEIRA, 2015; ASSIS, 2012; ALBUQUERQUE, 2018; PEREIRA, 2016), bem como os dois trabalhos apresentados em congresso (CHALHUB; GOMES, 2018; CHALHUB; BENCHIMOL; ROCHA, 2015), cujas autoras atuam em universidades da região Sudeste e uma na região Norte. Dentre os seis artigos considerados, os pesquisadores de quatro deles atuam na região sudeste (CHALHUB, 2014, LEÃO; SOFIATO; OLIVEIRA, 2017; SILVA; ROJAS; TEIXEIRA, 2015; SANTOS; ALVIM, 2014), um artigo foi escrito por pesquisadores do Sul (SOARES et al., 2013) e o outro foi escrito por pesquisadores da região Centro-oeste e Nordeste (ARAUJO; ALVES, 2017). Uma monografia e o Memorial 


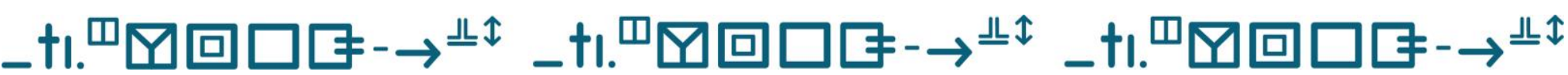

O uso da Língua Brasileira de Sinais para a promoção da inclusão em museus: revisando a literatura

Gabriela Sehnem Heck • José Luis Schifino Ferraro

Narrativo-descritivo foram escritos na região Nordeste (SILVA, 2015; OLIVEIRA, 2018) e a outra monografia (TORRES, 2012) é da região sudeste.

Os primeiros três anos da busca, 2009, 2010, 2011 não retornaram resultados. O ano de 2012 retornou dois documentos e nos anos de 2013, 2014 e 2016, considerou-se um documento. Com três documentos, os anos de 2015 e 2018 incluíram resultados de ambas as bases de dados. O ano com a maior densidade de publicações foi 2017 , com sete resultados.

O objetivo do levantamento de dados sobre atividades de Libras em museus foi buscar inspiração e ideias que permitam sua aplicação nos demais espaços museais, aumentando a acessibilidade e inclusão do público surdo. Porém, antes de pensar na elaboração de atividades inclusivas, utilizando a Libras como facilitador da comunicação e da aprendizagem em museus, deve-se observar se há elementos de acessibilidade nesses locais. Dessa forma, Chalhub, Benchimol e Rocha (2015, p. 4) sugerem uma lista para a identificação de acessibilidade aos surdos, especificamente em museus, levando em consideração alguns aspectos como:

presença de funcionários fluentes na Língua Brasileira de Sinais (LIBRAS) na recepção; presença de intérpretes guias para surdos nas exposições; presença de símbolos internacionais de informação; videoguia (LIBRAS e legendado) explicativo sobre a 


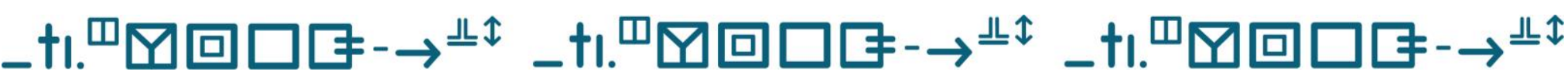

O uso da Língua Brasileira de Sinais para a promoção da inclusão em museus: revisando a literatura

Gabriela Sehnem Heck • José Luis Schifino Ferraro

\begin{abstract}
exposição; presença de SignWriting (escrita de língua brasileira de sinais) explicativo sobre o acervo exposto; presença de QR code em obras do acervo com tradução LIBRAS (oferecer o celular, caso visitante não tenha o aparelho adequado); exposições com informações em linguagem clara e coesa, preferencialmente em tópicos; exposições com informações apresentadas em diferentes recursos (sonoros, visuais, escritos, tácteis etc.); exposições com informações em tamanho e local adequados para leitura.
\end{abstract}

Alguns desses aspectos não exigem grandes alterações na exposição. Por exemplo, para uma educação mais adequada para surdos, as autoras destacam atividades que utilizem todos os recursos visuais disponíveis na exposição, atrelados à Libras, exposição de filmes legendados e o uso de QR Code, instalados em qualquer local da exposição, para disponibilizar vídeos em Libras dos materiais expostos (CHALHUB; BENCHIMOL; ROCHA, 2015). Porém, outros aspectos dependem de uma adaptação física do local, como a disponibilidade de uma iluminação adequada, para facilitar a visualização do intérprete e a realização de leitura labial, a utilização de recursos multissensoriais nas exposições, combinando imagens, maquetes, relevos e reprodução de objetos originais, além de técnicas de audiodescrição e audioguias (CHALHUB; BENCHIMOL; ROCHA, 2015).

De um modo geral, a maioria dos trabalhos considerados nessa revisão assumem os itens acima citados 


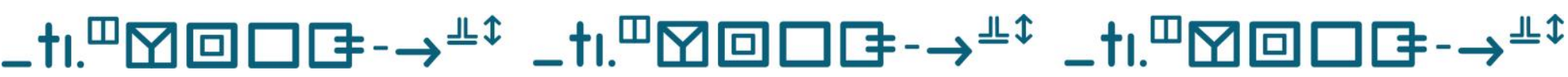

O uso da Língua Brasileira de Sinais para a promoção da inclusão em museus: revisando a literatura

Gabriela Sehnem Heck • José Luis Schifino Ferraro

como fundamentais para permitir a acessibilidade e, dessa forma, a realização de atividades inclusivas nos museus. Oliveira (2015) salienta que a presença de um educador surdo é essencial para a realização de qualquer atividade em Libras voltada à visitantes surdos ou ouvintes, visto que a presença deste educador nesses espaços permite a aproximação da comunidade surda ao museu, o que provoca uma transformação no olhar e permite o desenvolvimento do senso crítico desse grupo. A autora ainda destaca alguns critérios que definem a escolha desse profissional, considerando principalmente seu conhecimento de Libras e da cultura surda locais.

Assis (2012) destaca a presença de educadores surdos na integração da equipe que atende os visitantes, voltado ao atendimento em Libras, e propõe que essa visita ocorra mediante agendamento, para uma melhor organização do espaço. Santos e Alvim (2014) sugerem para o público com deficiência auditiva a oferta de monitores habilitados em Libras, para realizar um atendimento especializado.

Ainda na dissertação de mestrado de Oliveira (2015), a autora cita vários modos de aumentar a acessibilidade nos museus, para, enfim, elaborar atividades para a comunidade surda. A autora afirma que o museu, como promotor de conhecimento, pode desenvolver atividades de formação especializada de seus funcionários, voltada ao atendimento de público surdo. Um dos modos mais indicados para o atendimento desse visitante é a presença de mediadores 


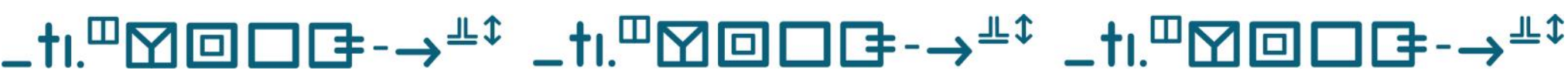

O uso da Língua Brasileira de Sinais para a promoção da inclusão em museus: revisando a literatura

Gabriela Sehnem Heck • José Luis Schifino Ferraro

capacitados em Libras, seja ele surdo ou ouvinte. Atendendo a uma demanda das políticas de inclusão, os museus podem também promover cursos de formação básica em Libras para seus funcionários de um modo geral.

Além de atuar na exposição, Oliveira (2015) sugere a criação de uma coordenadoria específica para o desenvolvimento dessas atividades de inclusão, podendo ser promovida por um educador surdo. Dessa forma, é possível elaborar visitas guiadas utilizando a Libras local, promover ciclos de palestras ou conferências, acompanhadas por um intérprete de Libras, e a apresentação de filmes com legenda e filmes voltados especificamente para o público surdo. Como sugestão da autora, essa coordenadoria poderia estar responsável por promover projetos de acessibilidade, elaboração de materiais multissensoriais, formação de educadores de diversas instituições e o estabelecimento de uma rede de encontro com professores para capacitação.

Oliveira (2015) também destaca que, com o desenvolvimento da tecnologia, é possível desenvolver recursos de tecnologia assistiva em Libras, por meio de visitas guiadas em videoguias, com sinalização em Libras e legenda na língua local, de forma a auxiliar a visitação de surdos quando não há um funcionário capacitado para atendê-lo. Chalhub, Benchimol e Rocha (2015) sugerem que os museus utilizem guias que apresentem o conteúdo informacional na língua de sinais, e pode-se também adotar um vídeo guia portátil com a informação do museu e do acervo de forma a 


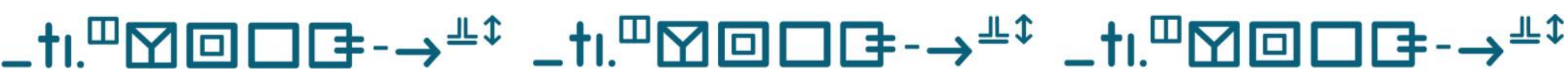

O uso da Língua Brasileira de Sinais para a promoção da inclusão em museus: revisando a literatura

Gabriela Sehnem Heck • José Luis Schifino Ferraro

dar autonomia ao visitante. Caso o visitante queira realizar uma visita autônoma, Assis (2012) sugere a elaboração de um guia de visitação impresso, com expressões reduzidas e vocabulário simplificado, além da disposição de signoguias ou videoguias. A utilização desses guias também é sugerida por Torres (2012), possibilitando às pessoas visitar a exposição utilizando a Libras com gravações de visitas guiadas e orientadas pelos mediadores e entrevistas com os curadores, todas interpretadas em Libras. Santos e Alvim (2014) também sugerem a realização de visitas guiadas com o uso de videoguia.

Silva (2015) acredita que fazer uso das Tecnologias da informação e comunicação (TICS) é uma forma de democratização da educação, "pois estas propiciam, sobretudo, a interação e, consequentemente, aprendizagem" (SILVA, 2015, p. 46). Visto que atualmente as TICs são muito utilizadas pelos surdos, a autora desenvolveu um museu virtual como proposta pedagógica para tornar as visitas mais participativas. O Museu Virtual possibilita experiências individuais para cada pessoa, também por meio de visitas virtuais guiadas, promovidas por um educador, respeitando a singularidade de cada visitante.

Chalhub e Gomes (2018, p. 5821) salientam a relevância do avanço dos recursos tecnológicos, como vídeos em Libras, representando "recursos que não interferem no acervo e ficam à disposição do público, não exigindo nenhum agendamento nem utilização de equipamento de difícil 


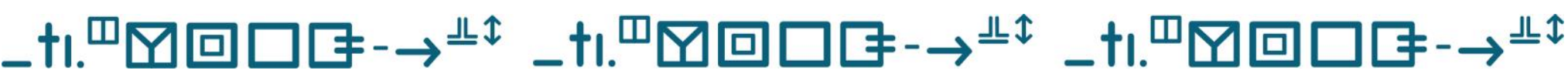

O uso da Língua Brasileira de Sinais para a promoção da inclusão em museus: revisando a literatura

Gabriela Sehnem Heck • José Luis Schifino Ferraro

aquisição". Chalhub, Benchimol e Rocha (2015) também destacam as TICs para a promoção de atividades e transmissão de conhecimento, indo em direção a um museu efetivamente inclusivo.

Segundo Oliveira (2018), os museus têm vários meios de inserir o visitante surdo em suas atividades, e um destes é a utilização da "Janela em Libras", um dispositivo que contém o conteúdo de determinado vídeo, traduzido em Libras, que fica disponível no canto inferior direito da tela. Salienta também a utilização de QR code para facilitar a compreensão e participação em atividades no museu. Independente dessas alternativas, é essencial a presença de um intérprete de Libras para a realização de qualquer atividade.

Além da nítida necessidade de adaptação da estrutura do museu, bem como capacitação de seus funcionários e elaboração de estratégias de inclusão e acessibilidade, os trabalhos revisados também trouxeram descrição de atividades que são realizadas ou que poderiam ser realizadas nos museus do Brasil, podendo ser adaptadas ao mundo. Oliveira (2015) sugere que as atividades voltadas aos visitantes surdos poderiam ocorrer em dias específicos da semana, com a presença de um educador capacitado, que as realizaria em Libras. Dessa forma, a comunidade surda saberia que em determinado dia da semana haveria atividades acessíveis a eles.

Essas atividades podem ser realizadas durante uma semana específica do ano, voltada à comunidade surda, 


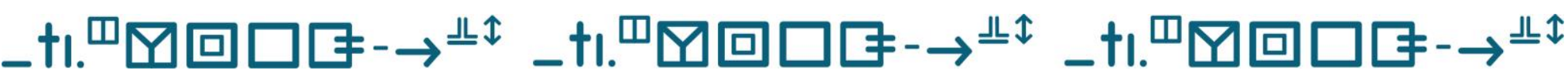

O uso da Língua Brasileira de Sinais para a promoção da inclusão em museus: revisando a literatura

Gabriela Sehnem Heck • José Luis Schifino Ferraro

utilizando a Libras, como sugerido por Oliveira (2015). Assim, evidencia-se o propósito da conscientização da condição do surdo na sociedade, por meio de contação de histórias, até visitas educativas, performances, oficinas de sinais, poesias, entre outros (OLIVEIRA, 2015). Essa semana voltada à comunidade surda poderia ser promovida em diversos centros culturais no mesmo período do ano como forma de divulgação da ciência e da cultura surda. De forma semelhante, Torres (2012) relata o desenvolvimento de uma semana cultural de conscientização dos sinais na arte, como forma de comemorar o Dia Internacional do Surdo, realizando uma série de atividades em Libras, fazendo parte do calendário cultural da região.

O estabelecimento de roteiros de visitas guiadas a acervos permanentes e temporários, segundo Oliveira (2015), permite que os visitantes possam participar ativamente das atividades promovidas pelos museus, e a realização de atividades práticas que envolvam a Libras são outros exemplos de atividades de fácil elaboração nesses locais. A autora observa que a realização de visitas temáticas com os educadores surdos ou intérpretes de língua de sinais, em dias e horários específicos, também são atividades que podem promover a inclusão.

Como citado por Oliveira (2015), na exposição é possível desenvolver atividades de contação de histórias em Libras por funcionários surdos, utilizando a exposição como recurso visual e educativo. As atividades podem trabalhar com 


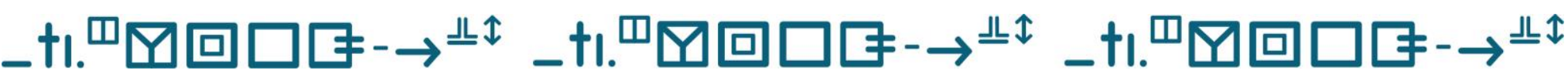

O uso da Língua Brasileira de Sinais para a promoção da inclusão em museus: revisando a literatura

Gabriela Sehnem Heck • José Luis Schifino Ferraro

feitas projeções de imagens conforme as batidas da música e a emissão de aromas, para uma experiência completa e inclusiva.

De forma semelhante, o projeto descrito por Torres (2012) corresponde a um espetáculo que envolve música, poesia e narração de histórias, interpretado em Libras. Além deste, existe outro - também referenciado pelo autor voltado para a formação em arte de jovens surdos e ouvintes, permitindo sua atuação como educadores em museus, por meio da Libras. A ideia é a de que esses jovens possam formar um coletivo artístico para discutir a cultura e identidade surda, fazendo uso de documentários e produzindo intervenções e performances (TORRES, 2012).

Outra atividade multissensorial é destacada por Araújo e Alves (2017), em que cada visitante recebe um aparelho que provê audiodescrições, adaptações visuais, diálogos e comentários durante o percurso da visita e passagem pelas exposições. Outra atividade que pode ser realizada é a exposição de documentários legendados, com o texto segmentado, como uma forma eficiente de compreensão pelo visitante surdo. Silva (2015) conclui que atividades que façam uso de dispositivos móveis, como smartphones, tablets, notebooks, e de seus aplicativos, são capazes de contribuir para o ensino e a aprendizagem dos visitantes surdos.

Chalhub (2014) salienta a importância da presença de vídeos em Libras, junto a legendas traduzidas, para permitir 


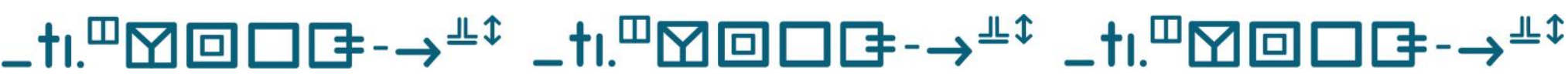

O uso da Língua Brasileira de Sinais para a promoção da inclusão em museus: revisando a literatura

Gabriela Sehnem Heck • José Luis Schifino Ferraro

a realização de visitas voltadas aos visitantes surdos, e propõe atividades na exposição que explorem mais a visualidade, a inserção dos vídeos e outras estratégias visuais como ferramenta pedagógica, ou para atividades de interação e comunicação. Para que ocorra a acessibilidade, os materiais audiovisuais devem ser legendados, em texto e em Libras, com volume controlável pelo visitante e fornecer serviços de transcrição em texto de exposições orais (CHALHUB, 2014).

Soares et al. (2013), por sua vez, assumem a necessidade de atrelar imagens com informações linguisticamente significativas, possibilitando a "acessibilidade aos bens culturais e imateriais do patrimônio brasileiro a essa parcela da população, seja por vídeos, por servidores fluentes em Libras ou da disponibilidade de intérpretes de Libras Português" (SOARES et al., 2013, p. 9). Do contrário, os visitantes acabam privados do direito ao lazer, à informação, à educação e à cultura. Chalhub e Gomes (2018) corroboram com essa ideia ao afirmar que as atividades devem estimular a capacidade visual, espacial e de compreensão do visitante surdo, a partir das exposições, permitindo ao visitante encontrar respostas a questões bastante complexas, de forma independente.

As atividades a serem realizadas podem utilizar informes visuais, a exemplo de fotografias, quadros com qualidade na resolução, livros ilustrativos, entre outros, pois “o trabalho com a imagem nos espaços museais com 


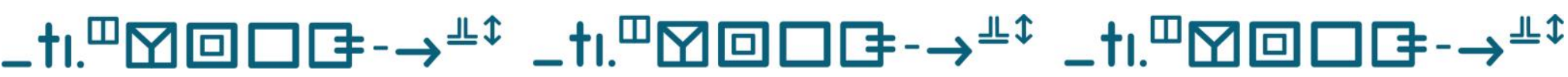

O uso da Língua Brasileira de Sinais para a promoção da inclusão em museus: revisando a literatura

Gabriela Sehnem Heck • José Luis Schifino Ferraro

visitantes surdos adquire outra dimensão, considerando-se o acesso às múltiplas linguagens e a mediação que geralmente é realizada por um profissional da área" (LEÃO; SOFIATO; OLIVEIRA, 2017, p. 61; OLIVEIRA, 2018). Além de auxiliar no ensino e aprendizagem, o uso de recursos visuais auxilia no processo de alfabetização e letramento de alunos, permitindo a realização de possíveis atividades inclusivas nos museus, aprimorando práticas pedagógicas que envolvam os visitantes surdos em diferentes espaços educacionais (LEÃO; SOFIATO; OLIVEIRA, 2017).

As atividades realizadas nos museus devem apostar na curiosidade como estímulo inicial, com foco em recursos visuais até ao estabelecimento de um elo com a vida cotidiana dos sujeitos, importante para manter o envolvimento e o interesse (CHALHUB; BENCHIMOL; ROCHA, 2015). Além disso, essas atividades devem, por fim, evidenciar a relação entre a exposição e os assuntos que os preocupam no dia a dia.

Segundo Albuquerque (2018), os museus têm a responsabilidade de reconstituição e preservação da memória surda, podendo ser realizados encontros formativos sobre história e cultura surda, voltados tanto para surdos, quanto para ouvintes (SILVA, 2015). Albuquerque (2018) salienta a importância da execução de atividades que envolvam visitas educativas, disponibilizando um intérprete de Libras. Araújo e Alves (2017) corroboram com o argumento, destacando que, para que a realização de qualquer atividade no museu seja facilitada, se destaca a 


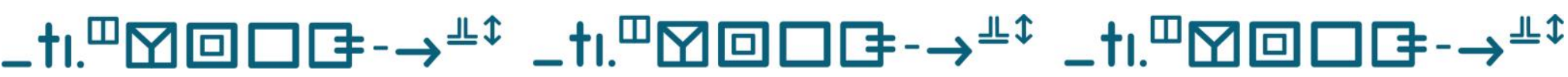

O uso da Língua Brasileira de Sinais para a promoção da inclusão em museus: revisando a literatura

Gabriela Sehnem Heck • José Luis Schifino Ferraro

importância da tradução em vídeo para a Libras, feita por um Tradutor e Intérprete de Libras (TILS), ficando a disposição em um quadro no canto inferior direito da tela, exibido simultaneamente à programação (ARAÚJO; ALVES, 2017; CHALHUB; GOMES, 2018).

Por fim, Oliveira (2015) salienta que, além dos recursos de tecnologia assistiva e da presença do intérprete de Libras, a presença de um educador surdo é fundamental para um bom atendimento, pois este possui o conhecimento da língua e cultura desta comunidade. Sendo assim, a possibilidade de visitar o museu e ter contato direto com a obra de arte significa para o surdo ter a possibilidade de:

Comunicação em Língua Brasileira de Sinais; Conhecimento cultural; Ampliação do vocabulário em Libras e em português; Aprendizagem visual: significado e interpretação das imagens; Visão crítica individual e em grupo: análise, opinião, diálogo e discussão (OLIVEIRA, 2015, p. 72).

\section{Análise de resultados}

Após a leitura e análise dos documentos selecionados, foi possível perceber que nove trabalhos foram produzidos na região sudeste, sendo esta com a maior densidade de publicações, seguida da região nordeste, com três trabalhos publicados. Nas regiões Norte, Centro-oeste e Sul, cada um retornou apenas um trabalho, conforme a Figura 1. Embora todas as regiões do Brasil estejam representadas nos resultados encontrados, destaca-se a região sudeste, em 


\section{_tı.}

O uso da Língua Brasileira de Sinais para a promoção da inclusão em museus: revisando a literatura

Gabriela Sehnem Heck • José Luis Schifino Ferraro

especial os estados de Rio de Janeiro e Minas Gerais: cada um com quatro documentos recuperados.

Figura 1 - Distribuição em percentual das regiões do Brasil onde os estudos foram realizados

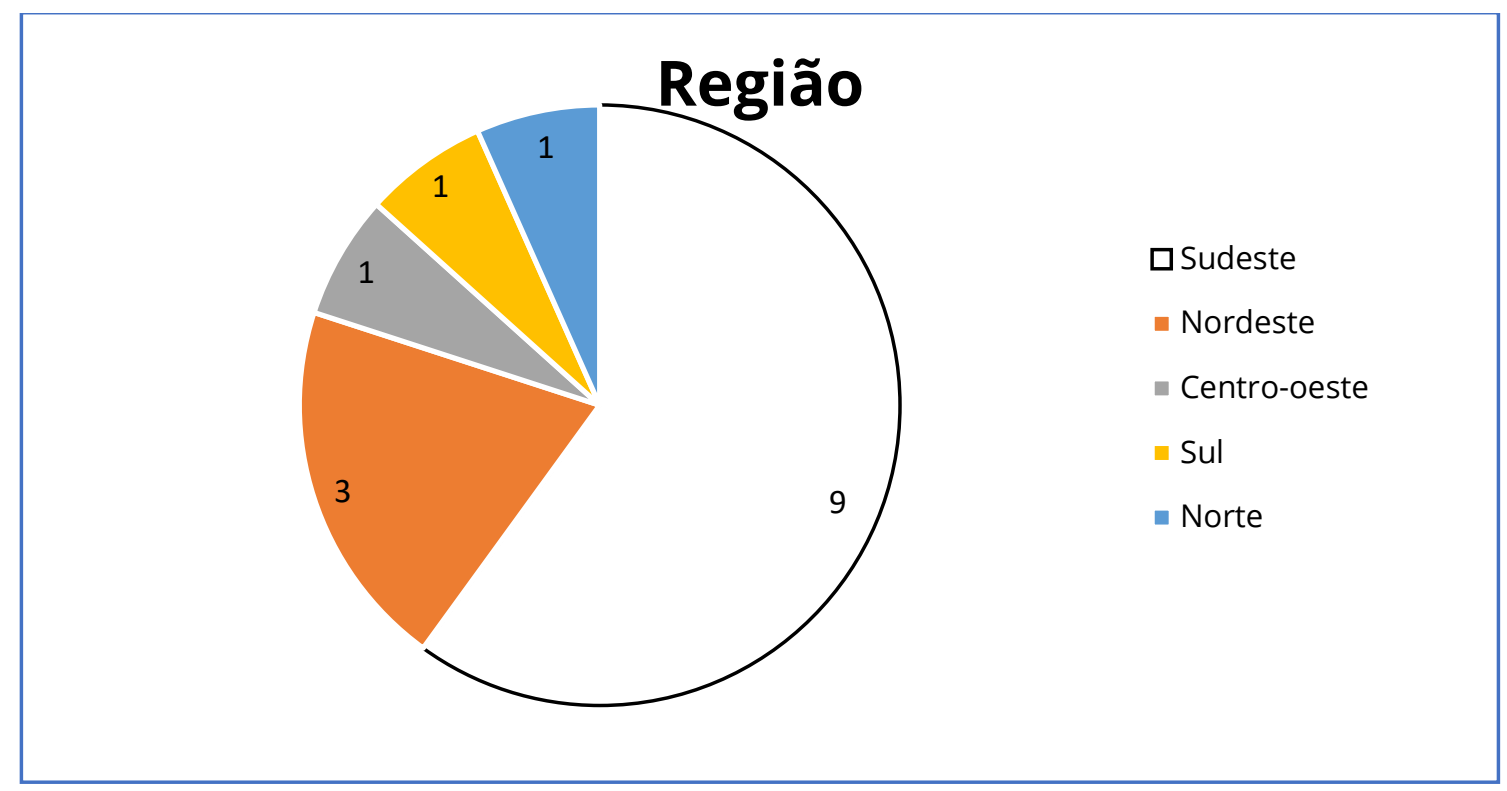

Fonte: Os autores (2020).

Descrição da imagem: Figura informativa, elaborada pelos autores, que apresenta a distribuição em densidade onde as pesquisas foram identificadas nas cinco regiões do Brasil: nove pesquisas no Sudeste, três pesquisas no Nordeste e uma pesquisa no Centro-Oeste, no Sul e no Norte, respectivamente.

Na Figura 2, estão apontadas as universidades e centros de pesquisa nas quais os autores dos documentos atuam, com destaque para a Universidade de São Paulo, em São Paulo, com cinco autores vinculados. 


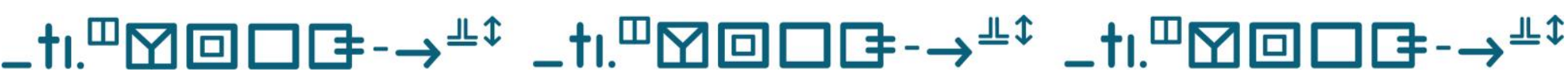

O uso da Língua Brasileira de Sinais para a promoção da inclusão em museus: revisando a literatura

Gabriela Sehnem Heck • José Luis Schifino Ferraro

Figura 2 - Número de autores vinculados às universidades e centros de pesquisa que sediaram a produção das investigações realizadas

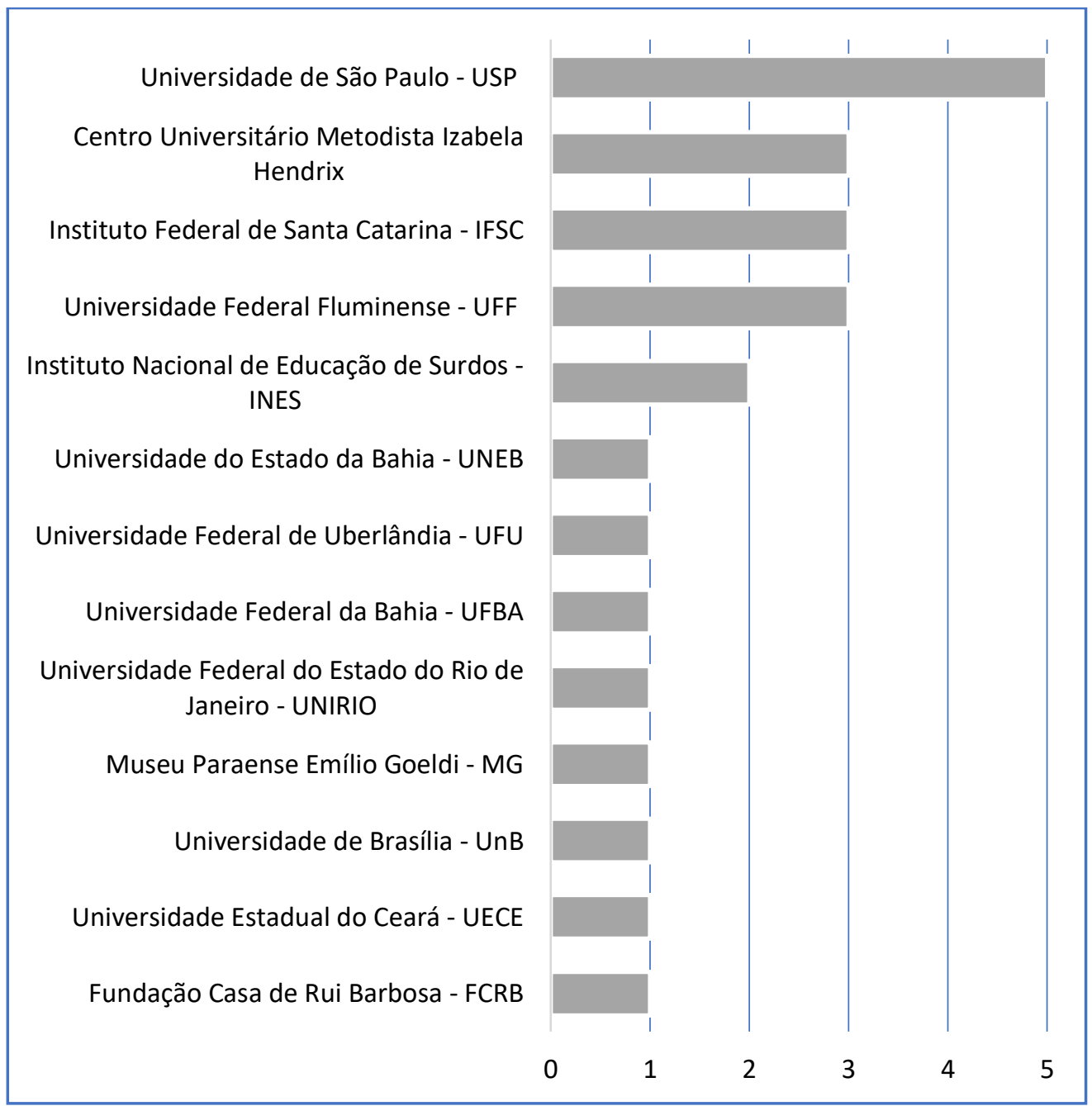

Fonte: Os autores (2020).

Descrição da imagem: Figura informativa, elaborada pelos autores, com dados sobre o número de autores e suas produções vinculadas às diversas universidades e centros de pesquisas dos trabalhos investigados.

Os resultados obtidos pela análise dos documentos encontrados foram agrupados por tipo de recurso utilizado, diferenciando-os em recursos humanos, quando as atividades realizadas sofrem ação direta de pessoas que 


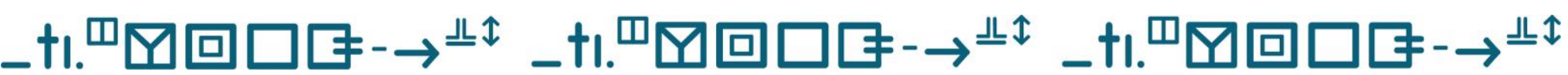

O uso da Língua Brasileira de Sinais para a promoção da inclusão em museus: revisando a literatura

Gabriela Sehnem Heck • José Luis Schifino Ferraro

atuam como mediadores, professores, monitores, educadores, entre outros, realizando atendimentos especializados, capacitações, auxílios em visitações e atividades interativas; em recursos estruturais, visto que há necessidade de criação ou alteração de espaços e exposições que peritam a realização das atividades; e recursos visuais, que utilizam a visualidade como apoio educacional, por meio de dispositivos eletrônicos, de mídias, como vídeos e imagens, apoio tecnológicos, fazendo uso de TIC's e outras ferramentas, materiais físicos que contenham informações visuais, claras e acessíveis e, por fim, eventos que também partem da visualidade para permitir a percepção e inclusão.

Além destes, distinguiu-se em recursos virtuais as atividades que fazem uso da internet, permitindo amplo acesso e facilidade, e a utilização de jogos como recurso lúdico e recreativo. A partir desses resultados, foi possível elaborar um quadro com uma síntese de todas as atividades reunidas (Quadro 1). 


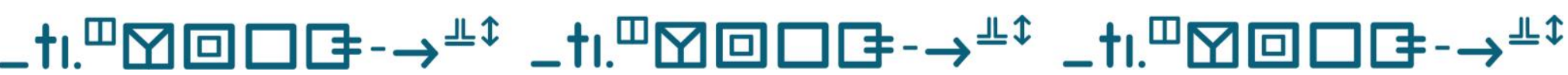

O uso da Língua Brasileira de Sinais para a promoção da inclusão em museus: revisando a literatura

Gabriela Sehnem Heck • José Luis Schifino Ferraro

\begin{tabular}{|c|c|c|}
\hline & $\begin{array}{l}\text { Guias com o conteúdo informacional na } \\
\text { língua de sinais; }\end{array}$ & $\begin{array}{l}\text { Chalhub; Benchimol; Rocha } \\
\text { (2015); Assis (2012); }\end{array}$ \\
\hline & $\begin{array}{l}\text { Roteiros de visitas guiadas à acervos } \\
\text { permanentes e temporários; }\end{array}$ & Oliveira (2015) \\
\hline Eventos & $\begin{array}{l}\text { Realização de um evento } \\
\text { multissensorial para surdos e ouvintes } \\
\text { que envolve música, poesia e narração } \\
\text { de histórias, interpretado em Libras; }\end{array}$ & Pereira (2016), Torres (2012) \\
\hline Virtuais & Museu virtual e visitas virtuais guiadas; & Silva (2015) \\
\hline Jogos & $\begin{array}{l}\text { Jogos de associação de palavras e } \\
\text { sinalização em Libras, Jogo Perfil ou } \\
\text { Fichas com imagens que estimulem os } \\
\text { visitantes a descobrir o que a imagem } \\
\text { que está na ficha representa; }\end{array}$ & Oliveira (2015) \\
\hline
\end{tabular}

Fonte: Os autores (2020).

Descrição da imagem: Quadro informativo, elaborado pelos autores, que possui dados sobre as atividades reunidas a partir dos documentos analisados, com seus recursos, suas atividades, suas caracterizações e seus respectivos autores.

\section{Considerações finais}

A partir da revisão literária empreendida, chama a atenção - de um modo geral - a indispensável presença de mediadores e funcionários surdos em museus e espaços culturais, como importante condição tanto para a elaboração de atividades para pessoas com deficiência, quanto pela visibilidade necessária, capaz de dar maior representatividade a uma comunidade por muito tempo silenciada. Por ter sido citada diversas vezes no decorrer desse trabalho, essa questão se demonstra fundamental para um atendimento acessível, inclusivo e representativo da comunidade surda, bem como na elaboração de atividades 


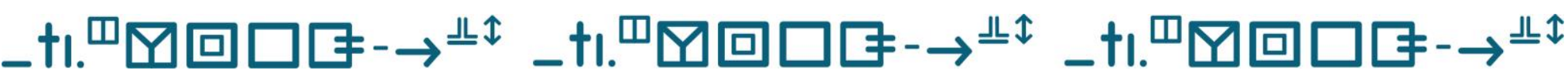

O uso da Língua Brasileira de Sinais para a promoção da inclusão em museus: revisando a literatura

Gabriela Sehnem Heck • José Luis Schifino Ferraro

significativas em museus, por meio da língua brasileira de sinais.

O primeiro ponto a ser discutido envolve a realização de atendimentos especializados por pessoal capacitado, visto que essa se configura como importante atividade de inclusão da comunidade surda em espaços que não estão adaptados a ela. A partir disso, os trabalhos analisados convergem na percepção sobre a importante presença de educadores, mediadores ou monitores, surdos ou ouvintes, capacitados em Libras, para a realização da tradução e interpretação da exposição para uma visita individual ou em grupo. Tal iniciativa vai ao encontro da Lei Brasileira de Inclusão da Pessoa com Deficiência, a Lei $n^{\circ} 13.146$ de 06 de julho de 2015, ao garantir o acesso aos bens culturais, atividades culturais e a locais de importância cultural cujos espaços oferecem eventos culturais acessíveis (BRASIL, 2015, Art. 42).

Para que haja pessoal capacitado para esse tipo de atendimento, se faz fundamental atividades de formação e capacitação especializada, com o fornecimento de cursos de formação básica em Libras e a realização de palestras sobre conscientização acerca da surdez e da comunidade surda, voltada para funcionários e para a comunidade em geral. Dessa forma, será possível realizar visitas temáticas e guiadas utilizando Libras, realizando atividades na exposição que explorem mais a visualidade e atividades de contação de histórias e oficina em Libras. 


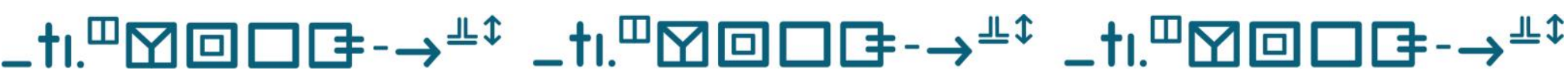

O uso da Língua Brasileira de Sinais para a promoção da inclusão em museus: revisando a literatura

Gabriela Sehnem Heck • José Luis Schifino Ferraro

Com relação à organização estrutural, sugere-se a criação de uma coordenadoria específica para assuntos que envolvam a acessibilidade e inclusão, permitindo o desenvolvimento de atividades significativas e especializadas, como sugerido por Oliveira (2015). Dessa forma, pode-se desenvolver atividades que utilizem TICs, como aplicativos, vídeoguias, signoguias, vídeos com legenda e com janela em Libras. Essas atividades exigem investimento em tecnologia e na produção dos materiais adaptados, necessitando de recursos, muitas vezes indisponíveis. A mesma coordenadoria seria responsável pelo desenvolvimento de materiais impressos, guias e roteiros de visitação, materiais multissensoriais e outros recursos que facilitem a visitação e a torne mais independente, permitindo uma maior autonomia ao visitante.

De modo geral, a realização de atividades voltadas à comunidade surda depende de determinados investimentos, alterações na exposição e na estrutura interna organizacional dos museus. Para tal, sugere-se o investimento sobre o desenvolvimento de projetos de pesquisa que estimulem as mudanças necessárias e tragam visibilidade para a comunidade surda e suas necessidades. Percebe-se também a importância das universidades públicas e federais, visto que dos 15 trabalhos selecionados para a construção da presente revisão bibliográfica, 13 foram produzidos nessas instituições, demonstrando a relevância das pesquisas públicas na área da educação de surdos e a importância do 


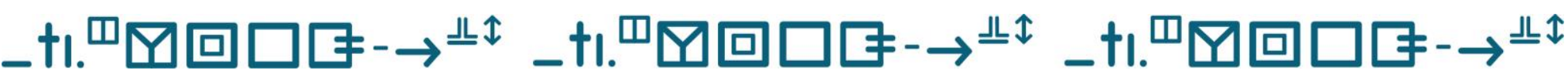

O uso da Língua Brasileira de Sinais para a promoção da inclusão em museus: revisando a literatura

Gabriela Sehnem Heck • José Luis Schifino Ferraro

investimento nesse tipo de pesquisa para a promoção da acessibilidade e inclusão em espaços culturais.

\section{Referências}

ALBUQUERQUE, ROBERTA SILVA VILARIÑO AGUILERA. AS VOZES E A MEMÓRIA DO SILÊNCIO:A IMPORTÂNCIA DA ATUAÇÃO DOS MUSEUS NA RECONSTITUIÇÃO E NA PRESERVAÇÃO DA MEMÓRIA SURDA. RIO DE JANEIRO. 2018. DISSERTAÇÃO (MESTRADO EM MEMÓRIA E ACERVOS) PPGMA, FUNDAÇÃO CASA DE RUI BARBOSA, RIO DE JANEIRO, 2018. DISPONÍVEL EM: HTTP://RUBI.CASARUIBARBOSA.GOV.BR/HANDLE/20.500.11997/7160. ACESSO EM: 07 JAN. 2020.

ARAÚJO, VERA LÚCIA SANTIAGO; ALVES, SORAYA FERREIRA. TRADUÇÃO AUDIOVISUAL ACESSÍVEL (TAVA): AUDIODESCRIÇÃO, JANELA DE LIBRAS E LEGENDAGEM PARA SURDOS E ENSURDECIDOS. TRABALHOS EM LINGÜístiCA APLICADA, V. 56, N. 2, P. 305, 2017. DISPONÍVEL EM: HTTP://SEARCH.PROQUEST.COM/OPENVIEW/248B5B04B6277F039617 4EC13B51 F52B/1?PQ-ORIGSITE=GSCHOLAR\&CBL=2040303. ACESSO EM: 07 JAN. 2020.

ASSIS, ELISA PRADO DE. ACESSIBILIDADE NOS BENS CULTURAIS IMÓVEIS:POSSIBILIDADE E LIMITES NOS MUSEUS E CENTROS CULTURAIS. 2012. DISSERTAÇÃO (MESTRADO EM ARQUITETURA E URBANISMO) FACULDADE DE ARQUITETURA E URBANISMO, UNIVERSIDADE DE SÃO PAULO, SÃO PAULO, 2012. DISPONÍVEL EM: HTTP://WWW.TESES.USP.BR/TESES/DISPONIVEIS/16/16133/TDE23052012-144557/PUBLICO/ELISA_PRADO_ASSIS_ME.PDF. ACESSO EM: 07 JAN. 2020. 


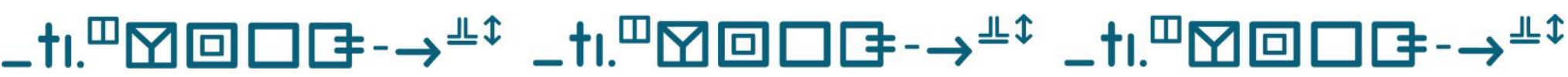

O uso da Língua Brasileira de Sinais para a promoção da inclusão em museus: revisando a literatura

Gabriela Sehnem Heck • José Luis Schifino Ferraro

BISOL, CLÁUDIA; SPERB, TANIA MARA. DISCOURSES ON DEAFNESS:

DISABILITY, DIFFERENCE, SINGULARITY AND CONSTRUCTION OF MEANING.

PSIC.: TEOR. E PESQ. VOL.26, N.1, PP.07-13, 2010.

BRASIL. LEI No 13.146, DE 6 DE JULHO DE 2015. INSTITUI A LEI

BRASILEIRA DE INCLUSÃO DA PESSOA COM DEFICIÊNCIA (ESTATUTO DA

PESSOA COM DEFICIÊNCIA). CÂMARA DOS DEPUTADOS. BRASílIA, 2015. DISPONÍVEL EM:

HTTPS://WWW.CNMP.MP.BR/PORTAL/IMAGES/LEI_BRASILEIRA_INCLUSAO_ _PESSOA_DEFICIENCIA.PDF. ACESSO EM 7 NOV. 2019.

CHALHUB, TANIA. ACESSIBILIDADE A MUSEUS BRASILEIROS: REFLEXÕES SOBRE A INCLUSÃO DE SURDOS. TENDÊNCIAS DA PESQUISA BRASILEIRA EM CIÊNCIA DA INFORMAÇÃO, V.7, N.2, 2014. ACESSO EM: HTTP://WWW.BRAPCI.INF.BR/INDEX.PHP/ARTICLE/VIEW/0000017428/5 8B18C5F0BDCFA08826A6ED04F7D4F16. ACESSO EM: 07 JAN. 2020.

CHALHUB, TANIA; BENCHIMOL, ALEGRIA; ROCHA, LUÍSA MARIA GOMES DE MATTOS. ACESSIBILIDADE E INCLUSÃO: A INFORMAÇÃO EM MUSEUS PARA OS SURDOS. IN: ENCONTRO NACIONAL DE PESQUISA EM CIÊNCIA DA INFORMAÇÃO, 16, 2015, JOÃO PESSOA. ANAIS. JOÃO PESSOA: UFPB, 2017. DISPONIVEL EM: HTTP://REPOSITORIOS.QUESTOESEMREDE.UFF.BR/REPOSITORIOS/HANDLE /123456789/2997. ACESSO EM: 07 JAN. 2020.

CHALHUB, TANIA; GOMES, MARISA. MUSEUS COMO ATIVIDADE EDUCATIVA: O QUE PENSAM OS ALUNOS SURDOS SOBRE ACESSIBILIDADE? IN:XIX ENCONTRO NACIONAL DE PESQUISA EM CIÊNCIA DA INFORMAÇÃO (XIX ENANCIB), 2018, LONDRINA, ANAIS. LONDRINA: UEL, 2018. DISPONÍVEL EM: HTTP://ENANCIB.MARILIA.UNESP.BR/INDEX.PHP/XIX_ENANCIB/XIXENAN CIB/PAPER/VIEW/1172. ACESSO EM: 07 JAN. 2020. 


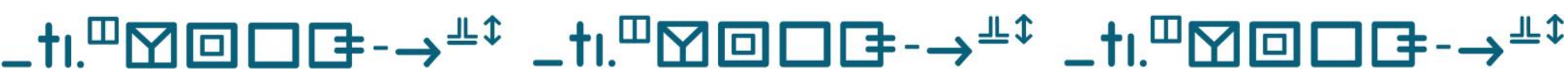

O uso da Língua Brasileira de Sinais para a promoção da inclusão em museus: revisando a literatura

Gabriela Sehnem Heck • José Luis Schifino Ferraro

\%20RAQUEL\%20PAIM\%20DE\%20OLIVEIRA.PDF. ACESSO EM 07 JAN. 2020.

PEREIRA, SARITA ARAÚJO. A UTILIZAÇão de TECNOLOGIA PARA

AMPLIAR A EXPERIÊNCIA SONORA/VIBRATÓRIA DE SURDOS. 2016. 116

F. DISSERTAÇÃO (MESTRADO EM ARTES) - UNIVERSIDADE FEDERAL DE UBERLÂNDIA, UBERLÂNDIA, 2016. DISPONÍVEL EM: HTTP://REPOSITORIO.UFU.BR/HANDLE/123456789/18027. ACESSO EM: 07 JAN. 2020.

QUADROS, RONICE MÜLLER DE. EDUCAÇÃO DE SURDOS: A AQUISIÇÃO DA LINGUAGEM. PORTO AlegRE: ARTMED, 1997.

SANTOS, JULIANA MOURTHÉ; ALVIM, MARINA NEIVA. EDUCAÇÃO INCLUSIVA EM ESPAÇOS NÃO-ESCOLARES: UM ESTUDO DE CASO DO MUSEU DAS MinAS E DO METAL EM BELO HORIZONTE, MG. ACERVO dA INICIAÇÃo CIENTífICA, BELO HORIZONTE, MINAS GERAIS, N. 1, 2014. DISPONIVEL EM: HTTPS://WWW.METODISTA.BR/REVISTASIZABELA/INDEX.PHP/AIC/ARTICLE/VIEW/696. ACESSO EM: 07 JAN. 2020.

SILVA, ALINE DA CRUZ PORTO. MUSEU VIRTUAL DA HISTÓRIA E MEMÓRIA DA COMUNIDADE SURDA: O CONTEXTO E O PERCURSO DE CRIAÇÃO. 86F. IL. 2015. MEMORIAL NARRATIVO-DESCRITIVO (MESTRADO PROFISSIONAL) - UNIVERSIDADE DO ESTADO DA BAHIA, SALVADOR, 2015. DISPONIVEL EM: HTTP://WWW.CDI.UNEB.BR/SITE/WPCONTENT/UPLOADS/2016/03/ALINE-DA-CRUZ-PORTO-SILVA.PDF. ACESSO EM: 07 JAN. 2020.

SILVA, JOÃo PAULO FERREIRA; ROJAS, ANGELINA ACCETTA; TEIXEIRA, GERLINDE AGATE PLATAIS BRASIL. ACESSIBILIDADE COMUNICACIONAL AOS SURDOS EM AMBIENTES CULTURAIS. CONHECIMENTO \& DiVERSIDADE, V. 7, N. 13, P. 103-115, 2015. DISPONÍVEL EM: HTTPS://WWW.REVISTAS.UNILASALLE.EDU.BR/INDEX.PHP/CONHECIMENTO 


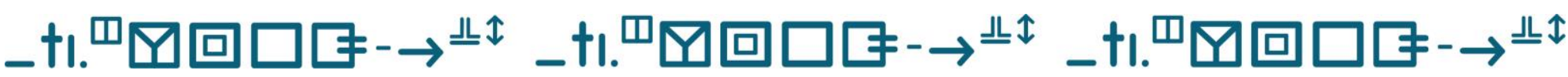

O uso da Língua Brasileira de Sinais para a promoção da inclusão em museus: revisando a literatura

Gabriela Sehnem Heck • José Luis Schifino Ferraro

_DIVERSIDADE/ARTICLE/VIEWFILE/1787/1427. ACESSO EM: 07 JAN. 2020.

SOARES, MARIA HELENA ALEMANY; PEREIRA, JANAÍ DE ABREU;

PATERNO, UÉSLEI, VINAS, ABENAMAR CRISTIAN ELCARAS. A INCLUSÃO DO SURDO NOS ESPAÇOS CULTURAIS E TURÍSTICOS DE FLORIANÓPOLIS.

REVISTA VIRTUAL DE CULTURA SURDA, N. 11, 2013. DISPONÍVEL EM: HTTPS://EDITORA-ARARAAZUL.COM.BR/SITE/ADMIN/CKFINDER/USERFILES/FILES/8)\%20PATERNO \%20\%26\%20CIA\%20REVISTA\%2011.PDF. ACESSO EM: 07 JAN. 2020.

TORRES, JULIANA GROTTI VIDAL. O DIREITO CULTURAL DA PESSOA COM DEFICIÊNCIA. 2012. TRABALHO DE CONCLUSÃO DE CURSO (GESTÃO DE PROJETOS CULTURAIS E ORGANIZAÇÃO DE EVENTOS) UNIVERSIDADE DE SÃO PAULO, SÃO PAULO, 2012. DISPONÍVEL EM: HTTP://CELACC.ECA.USP.BR/?Q=PT-BR/TCC_CELACC/DIREITO-CULTURALDA-PESSOA-DEFICIENCIA. ACESSO EM: 20 ABR. 2021.

\section{Publisher}

UNIVERSIDADE FEDERAL DE GOIÁS. CURSOS DE LETRAS: LIBRAS E DE LETRAS: TRADUÇÃO E INTERPRETAÇÃO EM LIBRAS/PORTUGUÊS DA FACULDADE DE LETRAS/UFG. PUBLICAÇÃO NO PORTAL DE PERIÓDICOS UFG. AS IDEIAS EXPRESSADAS NESTE ARTIGO SÃO DE RESPONSABILIDADE DE SEUS AUTORES, NÃO REPRESENTANDO, NECESSARIAMENTE, A OPINIÃO DOS EDITORES OU DA UNIVERSIDADE. 